\title{
SINTOMATOLOGIA DE DEPRESSÃO E SUPORTE FAMILIAR EM ADOLESCENTES: UM ESTUDO DE CORRELAÇÃO
}

\section{THE RELATION BETWEEN THE SYMPTOMS OF DEPRESSION AND FAMILY SUPPORT IN ADOLESCENTS}

Baptista MN, Oliveira AA. Sintomatologia de depressão e suporte familiar em adolescentes: um estudo de correlação. Rev Bras Cresc Desenv Hum 2004; 14(3): 53-59.

Resumo: A adolescência é marcada por diversas mudanças físicas/hormonais, sociais e psicológicas, podendo favorecer o desenvolvimento de diversos transtornos e sintomas mentais, como, por exemplo, a sintomatologia depressiva, sendo que a família pode influenciar o desencadeamento/manutenção de tais sintomas. O objetivo da pesquisa foi avaliar a correlação existente entre sintomatologia depressiva e a percepção de suporte familiar em estudantes do ensino médio. Foram participantes da pesquisa 154 adolescentes de uma escola pública. Os materiais utilizados foram um questionário de caracterização (contendo diversos fatores de risco para a depressão)^j-o Inventário de Depressão Infantil (CDI)) e o Parental Bonding Instrument (PBI), aplicados coletivamente em sala de aula. Os principais resultados demonstraram que quanto maior a simtomatologia depressiva no adolescente mais inadequado é a percepção do suporte familiar e quanto maior a freqüencia dos fatores de risco, maior é a simtomatologia depressiva no adolescente, estando o suporte familiar correlacionado negativamente com a simtomatologia depressiva nesta amostra.

Palavras chaves: Depressão. Adolescentes. Suporte familiar.

\section{INTRODUÇÃO}

Os principais sintomas da depressão estão relacionados com o humor deprimido, perda do interesse e prazer; energia reduzida, causando aumento da fatigabilidade e diminuição de atividades; concentração, atenção, auto-estimae autoconfiançareduzidas; idéias pessimistas do futuro; ideação suicida; alteração no sono e apetite diminuído, dentre outros ${ }^{1,2}$.

A depressão atualmente pode ser considerada um dos problemas mais comuns encontrados pelos profissionais na área de saúde mental ao diagnosticar e tratar seus pacientes. Os transtornos mentais e de comportamento correspondem a $12 \%$ das doenças mundiais ${ }^{3,4}$. Aproximadamente 15\% a20\% dos adultos já tiveram alguma sintomatologia depressiva durante suas vidas e, pelo menos $12 \%$ tiveram depressão em estado grave, a ponto de procurar ajuda psicoterapêutica e médica. A prevalência nas mulheres é duas vezes maior que nos homens, podendo chegar a $25 \%$, a partir da adolescência e os sintomas depressivos são responsáveis por cerca de 75\% das internações psiquiátricas $^{5}$. Além disso, 80\% das pessoas que tentam o suicídio estão com algum nível considerável de depressão no momento da tentativa ${ }^{6}$. A depressão tem incidência de $10 \%$ em pacientes do atendimento médico geral e chega a $15 \%$ dos pacientes intennados no hospital geral, apesar das pesquisas serem díspares em decorrência do tipo de amostra, instrumentos e metodologias diferenciadas ${ }^{7,8}$.

\footnotetext{
* Médico, Docente do Programa de Pós-Graduação Stricto-Sensu em Psicologia da Universidade São Francisco - ltatiba/ SP. Endereço para correspondência: Rua Dr. Miguel Pierro, 61 - Cidade Universitária 11- Barão Geraldo - Campinas/ SP - CEP I 30X3-300 - Tel 019)32S77224/97144972- E-mai1: daherbaptista@ig com br

** Psicóloga.
} 
$\mathrm{A} \mathrm{OMS}^{4}$ descreve diversos fatores que determinam a prevalência, o início e também a evolução dos transtornos mentais, dividindo-se em sociais e econômicos, como a pobreza que leva à privação econômica, ao baixo nível de instrução e ao desemprego. Outros fatores são os demográficos, como sexo e idade, somados à presença de doença física grave, pouco ou falta do suporte familiar, ameaças graves tais como conflitos e desastres. Os fatores estressantes também estão diretamente relacionados ao desencadeamento da depressão, isto é, uma criança e/ou adolescente que, por exemplo, vive em um ambiente hostil ou possui problemas familiares constantes, tende a aumentar as chances de desenvolver sintomatologia depressiva.

A depressão é um transtorno afetivo presente em todas as etapas do desenvolvimento; no entanto, somente há aproximadamente 40 anos é que este fenômeno vem sendo mais enfocado em crianças e adolescentes. O profissional da saúde e da educação deve estar atento aos sintomas de depressão em crianças e adolescente pois, assim, poderá detectar e diagnosticar diferencialmente tal transtorno, bem como propiciar tratamento adequados $^{9,10}$.

A adolescência pode ser considerada como o período da vida humana que sucede à infância, quando ocorre uma série de mudanças corporais, físicas, honmonais e psicológicas, podendo se tornar um processo conflituoso na medida em que se caracteriza por uma fase de auto-afirmação, absorção dos valores culturais e elaboração de projetos para inserção da integração social ${ }^{11.12}$.

PAPALIA e col..$^{13}$ relatam não haver um momento exato para o começo e o fim da adolescência; em termos cronológicos, pode ser considerado dos 12 aos 20 anos, sendo que, nas sociedades modernas, considera-se como seu início a capacidade de reprodução, ou seja, a menstruação para as meninas e a produção de esperma para os meninos. O final da adolescência pode estar ligado a diversos fatores, tais como o alistamento militar para os homens; relacionamentos duradouros e formação de família; escolha da carreira profissional ou independência financeira e também o amadurecimento do pensamento cognitivo e abstrato.

Dentre os diversos fatores sociais/culturais que estão envolvidos no desencadeamento de sintomatologia depressiva em crianças e adolescentes, pode-se considerar a família como fundamental como modelo de comportamentos ligados à estratégias de enfrentamento, bem como desenvolvimento de auto-regras relacionadas à autoestima da criança e adolescente ${ }^{14}$.

Nos dias atuais houve um grande crescimento das famílias formadas somente por mulhe- res e seus filhos, além da redução do tamanho da família (mudanças na estrutura familiar), observando-se que o número crescente de mulheres esteio da casa se deve a vários fatores, como a maior participação feminina no mercado de trabalho, igualdade nos direitos e deveres entre homens e mulheres, quebra de valores tradicionais como o casamento, alto índice de pobreza e crescente número de desemprego, gerando necessidade de aumento dos rendimentos ${ }^{15}$.

Baptista ${ }^{16}$ salienta a diferença entre estrutura e suporte familiar, sendo a estrutura familiar o número de pessoas de uma família e como se dispõe, ou seja, suas características físicas (ex. família é intacta ou pais separados ou até falecidos; quantas pessoas moram na casa). Já, o suporte familiar está relacionado aos aspectos psicológicos, como manifestação de carinho, atenção, diálogo, proximidade afetiva, liberdade, superproteção e independência existente entre os membros da família.

Segundo Herring e col. ${ }^{17}$, as relações familiares e a sintomatologia depressiva possuem relações bidirecionais, sendo que a família pode ser considerada como um modelo potente para o desenvolvimento de vínculos positivos e seguros nos adolescentes, bem como a sintomatologia depressiva no adolescente pode modificar a percepção e os vínculos com a família.

O objetivo principal desta pesquisa foi avaliar a correlação existente entre sintomatologia depressiva e a percepção de suporte familiar em estudantes do ensino médio, enquanto que os objetivos específicos foram avaliar a prevalência de sintomas depressivos na amostra; averiguar a prevalência de sintomas depressivos em ambos os sexos e correlacionar os fatores de risco para depressão com a prevalência de sintomatologia depressiva na amostra.

\section{MÉTODO}

\section{Participantes}

Foram participantes desta pesquisa 154 adolescentes, estudantes do ensino médio de uma escola estadual da cidade de Mogi Guaçú, interior de São Paulo, com idade distribuída da seguinte forma: 14 anos (representando $11 \%$ da amostra), 15 anos (40,9\% da amostra), 16 anos $(31,2 \%)$ e com 17 anos (16,9\%, da amostra), sendo que $50,6 \%$ estava cursando o $1^{\circ}$ ano do ensino médio, $26 \%$ o $2^{\circ}$ ano e $23,4 \%$ o $3^{\circ}$ ano.

O sexo feminino predominou, $72,7 \%$ da amostra, e os dados mostraram que $85,1 \%$ dos jovens residiam com sua família nuclear (pai, mãe e irmãos), 5,2\% moravam com outros (avôs, tios, 
primos), $8,4 \%$ somente com a mãe e 1,3\% somente com o pai, além do que $83,1 \%$ dos pais viviam juntos. Em relação à renda familiar, $52,7 \%$ da amostra relatou pertencerem à faixa entre 3 e 6 salários mnimos, seguidas de 22,6\% com renda entre 1 e 3 salários,18,3\% entre 6 e 9 salários e, por último, 6,5\% com rendimento acima de 10 salários mínimos, lembrando que 39,6\% dos participantes não respondeu a esta questão.

Em relação aos fatores de risco para desencadeamento de depressão, 3,9\% da amostra estudada possuía alguma doença crônica e $26 \%$ dos adolescentes tinham passado por algum evento estressante nos últimos três meses. Também nos últimos três meses, $97,4 \%$ dos estudantes não havia passado por alguma intervenção cirúrgica e, 85,7\% disseram não ter passado por experiência de luto. Em relação à ingestão de medicamento psiquiátrico, $97,4 \%$ relatou a não utilização destas substâncias e, 17,5\% da amostra apontou que na sua família nuclear havia alguém com depressão.

\section{Material}

Os instrumentos utilizados para a coleta de dados desta pesquisa foram:

Questionário de Caracterização - desenvolvido pelos pesquisadores, continha duas perguntas abertas, quatro perguntas fechadas e seis perguntas mistas a fim de se obter dados sóciodemográficos, médicos, familiares, pelo levantamento de fatores de risco relacionados com o desencadeamento de simtomatologia dèpressiva, tais como ingestão de medicamentos, prevalência de depressão em parentes de primeiro grau, luto e intervenções cirúrgicas e eventos estressantes nos últimos três meses, prevalência de doença crônica, falecimento e/ou separação de pais na infância e adolescência.

Parental Bonding Instrument (PBI) - este instrumento contém 25 perguntas do tipo likert (com 4 pontos) e foi desenvolvido por Paker, Tupling \& Brown ${ }^{18}$, tendo como objetivo medir quatro dimensões presentes no suporte familiar, dentre elas: superproteção; permissão de autonomia e independência (a dimensão foi intitulada de autonomia); rejeição e indiferença (indiferença) e carinho, sendo que o inventário foi traduzido e adaptado para o português, com o auxílio de um professor bilíngüe ${ }^{19}$.

\section{Inventário de Depressão Infantil}

[Children's Depression Inventory/CDI ${ }^{20}$ ] adaptado e normalizado por Gouveia e cols. na população de João Pessoa. O CDI demonstrou ser um instrumento com características psicométricas (validade, fidedignidade e normas) razoavelmen- te satisfatório para discriminar respondentes que apresentam traços referentes a provável depressão. O CDI ficou constituído de 20 itens que medem a gravidade da depressão em crianças e adolescentes, avaliam a disforia, o pessimismo, a auto-estima, o desprezo, o isolamento social, o rendimento escolar, a conduta social e sintomas vegetativos.

\section{Procedimentos}

Primeiramente, foi solicitada autorização da diretoria da escola para o desenvolvimento da pesquisa na instituição, sendo que seis classes foram sorteadas aleatoriamente na escola e entregues aos alunos um Termo de Consentimento Livre e Esclarecido (TCLE) com os objetivos da pesquisa, para a assinatura dos responsáveis e prosseguimento da aplicação dos instrumentos. O tempo de duração para a aplicação dos instrumentos variou entre 20 e 30 minutos e uma parte (37,39\%) das autorizações enviadas aos pais não voltarem, não sendo possível avaliar os motivos deste ocorrido.

A análise estatística inferencial foi realizada correlacionando-se os resultados do Inventário de Depressão Infantil (CDI) com o Instrumento de Avaliação das Relações Familiares (PBI) e com a somatória dos fatores de risco, utilizando-se o teste de correlação não paramétrico de Sperman, com nível de significancia $0,05 \%$, adequado para as ciências sociais; para avaliar a associação entre variáveis nominais com variáveis ordinais, foi utilizado o teste de Mann-Witney, sendo os dados analisados pelo programa Statistical Package for Social Science (SPSS - versão 10,0).

\section{RESULTADOS}

Em relação aos resultados do inventário de depressão na amostra, observou-se que nove adolescentes ultrapassaram o ponto de corte (escores acima de 18) estabelecido pelo instrumento, totalizando $5,6 \%$ da amostra total, sendo que $89 \%$ era do sexo feminino e $11 \%$ do sexo masculino.

Foram encontradas associações entre a pontuação do CDI e ter passado por eventos estressantes no período de três meses (U-1417,5; $\mathrm{p}=0,00)$; sintomatologia de depressão e ter alguém na família (pai, mãe) com depressão ( $U=1238,0$; $p=0,023)$; simtomatologia depressiva e estar tomando algum medicamento psiquiátrico $(\mathrm{U}=53,5 ; \mathrm{p}=0,005)$ e sintomatologia depressiva e ter pais falecidos $(U=208,0 ; p=0,008)$, não se encontrando a associação entre sintomatologia depressiva e ter pais separados. 
Tabela 1 - Correlação entre CDI, PBI e fatores de risco

\begin{tabular}{lcc}
\hline DIMENSÕES & $\begin{array}{c}\text { Valor da } \\
\text { Correlação }\end{array}$ & $\begin{array}{c}\text { Nível de } \\
\text { significância (p) }\end{array}$ \\
\hline Fator de risco x cdi & 0,231 & 0,004 \\
\hline Cdi x superproteção/independência & 0,250 & 0,002 \\
\hline Cdi x carinho/indiferença & 0,649 & 0,000 \\
\hline Cdi x pbi total & $-0,567$ & 0,000 \\
\hline Superproteção x indiferença & 0,211 & 0,009 \\
\hline Independência x carinho & 0,312 & 0,000 \\
\hline
\end{tabular}

Os principais eventos estressantes relatados pelos adolescentes foram: brigas com namorado ou fim de namoro (32\%); brigas e desavenças com a família (27\%); falecimento de entes queridos (familiares ou amigos), com 12\%; problemas pessoais/crise de identidade (7\%); respostas em branco (7\%); discussão com amigos (5\%); doenças na família (5\%) e mudança de cidade ou local de moradia (5\%).

A análise estatística entre o CDI e os fatores de risco demonstrou a existência de correlação, observando-se que a pontuação do inventário de depressão se correlacionou positivamente com os fatores de risco, apontando que quanto maior a pontuação dos fatores de risco, maior a pontuação de sintomatologia depressiva.

Entre a variável sintomatologia depressiva e superproteção/independência também foi observada correlação positiva, significando que quanto maior a frequência de sintomas depressivos, maior a pontuação em termos de superproteção familiar e falta de autonomia do adolescente frente aos pais.

Já em relação à correlação entre o CDI e as dimensões Carinho/Indiferença do PBI, observou-se que quanto maior a sintomatologia depressiva, menor a percepção de demonstração de expressão de emoções como carinho, afeto, elogios e suporte emocional da família em situações desconfortantes para o adolescente.

Em relação à comparação dos sintomas depressivos e o suporte familiar total, observouse correlação negativa e moderada entre estas duas variáveis, significando que quanto maior a sintomatologia depressiva, menor a percepção do suporte familiar.

Por último, detectou-se correlação entre a variável superproteção versus indiferença; isto significa que quanto maior a pontuação na superproteção, maior também é a pontuação na indiferença que os filhos sentem por parte de seus familiares. Na análise entre independência e carinho, foi observada a existência de correlação positiva, demonstrando que quanto malor a pontua- ção na dimensão carinho, também maior a pontuação na dimensão independência fornecida ao adolescente pela família.

\section{DISCUSSÃO}

A depressão vem se mostrando como um dos transtornos de maior prevalência na população em geral, inclusive sendo metaforicamente chamada como o "resfriado comum dos distúrbios psiquiátricos”22.

Apesar do instrumento de avaliação de depressão infantil (CDI) ser considerado um rastreamento, não se podendo afirmar o diagnóstico de depressão por meio de sua aplicação, observa-se que a prevalência de sintomatologia depressiva encontrado na atual pesquisa está de acordo com Baptista ${ }^{23}$ que utilizou uma amostra de 113 participantes estudantes do sexo feminino de Colégio e Magistério, observando-se 5,3\% da amostra acima do ponto de corte; no entanto, esses resultados divergem dos encontrados por Barbosa e cols. ${ }^{24}$, em uma pesquisa epidemiológica com 807 crianças e adolescentes na cidade de Bananeiras (Paraíba), totalizando 22\% da amostra, coletada em escolas públicas. Em relação a este ponto, é interessante notar que talvez a sintomatologia depressiva possa ser considerada como um conjunto de comportamentos e cognições esperadas para esta fase de desenvolvimento do adolescente.

Em relação à associação entre os eventos estressantes e a sintomatologia de depressão encontrada neste estudo, Oliveira e cols. ${ }^{25}$ apontam para esta relação importante, inclusive focalizando os transtornos psiquiátricos, e não apenas os transtornos de humor, sendo que os eventos de vida seriam potentes desencadeadores de sintomas psiquiátricos. Friis e cols. ${ }^{26}$, em uma pesquisa longitudinal com uma amostra comunitária de adolescentes e adultos jovens de 14 a 24 anos, encontraram os eventos negativos de vida e o suporte familiar como preditores do desencadeamen- 
to de novos episódios de depressão. Da mesma forma, Pine e cols. ${ }^{27}$, ao realizar um estudo longitudinal de seis anos com uma amostra de 776 jovens na cidade de Nova York, encontraram os eventos de vida na adolescência como preditores de sintomatologia depressiva na vida adulta.

Ao analisar a associação entre sintomatologia depressiva no adolescente e este possuir familiares com depressão, pode-se abordar o assunto de diversas formas. Por exemplo, sabe-se que as questões genéticas são importantes para explicar o desencadeamento de depressão, por herdabilidade; no entanto, os fatores ambientais também são fundamentais para o desencadeamento de sintomatologia depressiva, já que a presença de pais com sintomatologia depressiva em algum momento do desenvolvimento infantil e/ou adolescente pode propiciar a aprendizagem de modelos cognitivos negativos e o desenvolvimento de vínculos inseguros ${ }^{28-30}$.

A não associação entre sintomatologia depressiva e ter pais separados, encontrados nos atuais resultados, não corroboram os resultados de Canetti e cols. ${ }^{31}$, que encontraram associação entre pais separados e maior número de sintomas psiquiátricos nos adolescentes, menor sensação de bem-estar e percepção de baixo suporte provindo da família, especificamente menor carinho e maior controle dos pais, quando comparados com adolescentes que possuíam famílias intactas. Segundo Gonzáles-Forteza e cols. ${ }^{32}$, as relações familiares na adolescência são fundamentais para o estabelecimento de novas relações maduras na vida das pessoas, funcionando inclusive como modelos de relação para o adolescente. Diversas são as pesquisas que demonstram a importância das relações sociais e familiares como suportivas ou amortecedoras (buffering) nos transtornos psiquiátricos, ou mesmo correlações entre o inadequado suporte familiar e sintomatologia depressiva ${ }^{17,23.33,34}$.

Mesmo os princípios de aprendizagem envolvidos na díade pais-filhos são fundamentais para explicar a importância do padrão familiar como modelo para as novas relações que o adolescente estabelecerá durante a sua vida, como por exemplo, a modelagem e a modelação. Por exemplo, Bandura ${ }^{35}$ enfatiza a aprendizagem através dos processos vicários ou de modelação, em que o observador pode adquirir habilidades cognitivas e, conseqüentemente, novos comportamentos, por meio da observação dos padrões de comportamentos de outras pessoas.

Nesta mesma linha de pensamento, Miller e cols. ${ }^{36}$ examinaram a transmissão de padrões familiares entre gerações, especificamente entre mães e filhas, através de uma pesquisa longitudinal de 10 anos de acompanhamento. Pela análise de regressão logística, demonstrou-se que a transmissão do estilo parental se mostrou independente de sintomas depressivos da mãe ou da filha ou até mesmo do status sócioeconômico. Os resultados demonstraram que o estilo denominado de controle ou coação afetiva (altos níveis de carinho e de superproteção) pode ser transmitido de mães para filhas, podendo este estilo ser até um fator de risco para o desenvolvimento de depressão nas gerações vindouras. Os autores também apontaram a possibilidade do estilo de personalidade da filha (traços de comportamento) ser um fator que auxilia o desencadeamento de estilos parentais maternos específicos, tais como a irritabilidade da filha tender a suscitar o estilo de controle afetivo das mães.

Miller e cols. ${ }^{37}$ também apontam que apesar da relação entre mãe-filhos ser mais preditora do que entre pai-filhos.

Rey $^{44}$ também apontou uma associação entre depressão e carinho provindo da mãe, em 563 adolescentes depressivos como sendo a de maior magnitude $(t=-2,750 ; p=0,006)$. A regressão logística também demonstrou associação próxima do limite de significância entre depressão e carinho provindo do pai $(\mathrm{t}=-1,970$; $\mathrm{p}=0,05 \mathrm{l}$ ) e controle (superproteção) provindo da mãe $(t=-1,924 ; p=0,054)$.

\section{CONCLUSÃO}

Deste modo, os principais resultados demonstraram que quanto maior a sintomatologia depressiva no adolescente mais inadequada é a sua percepção do suporte familiar e quanto maior a freqüência dos fatores de risco, maior é a sintomatologia depressiva no adolescente, estando o suporte familiar correlacionado negativamente com a sintomatologia depressiva nesta amostra.

Especial cuidado deve ser tomado em relação às correlações encontradas neste estudo pois, como afirmam Klimidis e cols. $4^{45}$, grupos de indivíduos que possuem algum tipo de patologia tendem a perceber o seu suporte familiar como mais supercontrolador e fornecendo menores taxas de carinho, sendo que o contrário também é verdadeiro; portanto, esta relação não deve ser considerada como causal. Deve-se tomar igualmente especial cuidado quanto à generalização dos achados desta pesquisa dado que o número de participantes foi pequeno e a coleta ocorreu somente em escola pública. 


\begin{abstract}
In adolescence, there are many changes such as: physical, hormonal and psychological that could have a bearing on the development of an assortment of disorders and mental symptoms, for example depression symptomology, which the family can have an influence over by starting and maintaining the development of these symptoms. The objective of the research is to evaluate the correlation that exists between the depression symptomology and the perception of family support in high school students. One hundred and fifty-four (154) students from a public high school participated in the research. The materiais used were an identification questionnaire (with several risk factors in depression), the Children's Depression Inventory (CDI) Scale, and the Parental Bonding Instrument (PBI). The questionnaires were given collectively in the classroom. The main results showed that the more an adolescent exhibited a depressive symptomology, the more inadequate the adolescent's family support is, and thus a greater risk factor. In this sample of adolescents, the research can conclude that family support is negatively related to depressive symptomology.
\end{abstract}

Keywords: Depression. Adolescents. Family support.

\section{REFERÊNCIAS}

1 Caetano D, coordenador. Classificação de Trans tornos Mentais e de Comportamento da CID-10: Descrições Clínicas e Diretrizes. Organiz. Mund. da Saúde. Porto Alegre: Artes Médicas; 1993.

2 Americam Psychiatric Association. Diagnostic and statistical manual of mental disorders $4^{\text {th }}$ edi tion. Washington, DC: America Psychiatric Association; 2000. DSM-IV-TRIM - Manual Di agnóstico e Estatístico de Transtornos Mentais. Trad. Cláudia Dornelles. $4^{\mathrm{a}}$ ed. rev. Porto Alegre: Artmed; 2002.

3 Young JE, Beck AT, Weinberger A. Depressão. In: Manual clínico dos transtornos psicológicos. Porto Alegre: Art. med.; 1999. p.273312.

4 Organização Mundial da Saúde - Relatório sobre a Saúde no Mundo: Nova concepção, nova esperança; 2001.

5 Fennell MJV. A natureza da depressão. In: Tera pia cognitivo-comportamental para problemas psiquiátricos. São Paulo: Martins Fontes; 1997. p. 241-337.

6 Reinecke MA. Suicídio e depressão. In: Estratégias cognitivo-comportamentais para intervenção em crises. Campinas: Editorial Psy II; 1995. P 99-141.

7. Demétrio FN. Depressão: bases anátomo-fisiológicas. In: Sobre comportamento e cognição. São Paulo: Arbytes; 1997. p. 151-158.

8 Baptista, MN, Assumpção-Júnior, FB. Epidemiologia e gênero na depressão. In: Depressão na adolescência uma visão multifatorial, São Paulo: EPU; 1999. p.57-58.

9 Versiani M, Reis R, Figueira I. Diagnóstico do transtorno depressivo na infância e adolescência. Jornal Brasileiro Psiquiatria 2000; 10(12): 367-82.
10 Assumpção-Júnior FB, KuczynskiE. Infância e adolescência. In: Depressões em medicina interna e em outras condições médicas: depressões secundárias. São Paulo: Atheneu; 2000. p. 387-400.

11 Ferreira ABH, organizador. Novo dicionário da língua portuguesa. Rio de Janeiro: Nova Fronteira; 1986. p.46 e 755.

12 Bee H. Desenvolvimento fisico e cognitivo na adolescência. In: O ciclo vital. Porto Alegre: Artes Médicas; 1997. p. 318-324.

13 Papalia ED, Olds SW. Desenvolvimento físico e cognitivo na adolescência. In: Desenvolvimento humano. Porto Alegre: Artmed; 2000. p. 310.

14 Baptista, MN, Baptista, ASD, Dias, RR. Estrutura e suporte familiar como fatores de risco na depressão de adolescente. Psicologia Ciência e Profissão 2001; 21: 52-61.

15 Ribeiro RM, Sabóia AC, Branco HC, Bregman S. Estrutura familiar, trabalho e renda. In: Familia brasileira a base de tudo. Brasília: Cortez; 2000. p.143-145.

16 Baptista MN. Adolescência, depressão e suporte familiar. In: Depressão na adolescência uma visão multifatorial. São Paulo: EPU; 1999. p. 31-441.

17. Herring M, Kaslow NJ. Depression and attach ment in families: a child-focused perspective. Family Process 2002; 41 (3): 494-518.

18 Parker G, Hadzi-Pavlovic D. Parental representtions of melancholic and non-melan-cholic depressives: examining for specificity to depressive type and for evidence of additive effects. Psychological medicine 1992; 22: 657-65.

19 Baptista MN. Depressão e suporte familiar: perspectivas de adolescentes e suas mães [dissertação]. Campinas (SP): Pontifícia Universidade Ca tólicade Campinas; 1997. 
20 Kovacs M. The Children’s Depression Inventory: a self-rated depression scale for schoolaged young' sters. University of Pittsburh; 1983.

21 Gouveia W, Barbosa GA, Almeida HJF, Galão AA. Inventário de depressão infantil - CDI: estudo de adaptação com escolares de João Pessoa. Jornal Brasileiro de Psiquiatria 1995; 44: 345-9.

22 Yates MJ. Depressão profunda recorrente. In: Compreendendo a terapia cognitiva. Campinas: Editorial Psy; 1998.

23 Baptista MN. Sintomas depressivos em adoles centes e a percepção da estrutura familiar. Infanto - Revista Neuropsiquiatria da Infância e Adolescência 1998; 6: 34-40.

24 Barbosa GA, Dias MR, Galão AA, Lorenzo WCG. Depressão infantil: um estudo de prevalência com o CDI. Infanto - Revista Neuropsiquiatria da Infância e Adolescência 1996; 4: 36-40.

25 Oliveira MG, Fonseca PP, Del-Porto JA. Versão brasileira de Paykel para eventos de vida recentes. Revista Brasileira de Terapia Comportamental e Cognitiva 2002; 4: 47-61.

26 Friis RH, Wittchen HU, Pfister H, Lieb R. Life events and changes in the course of depression in young adults. Eur Psychiatry 2002; 17: 24153.

27 Pine DS, Cohen P, Johnson JG, Brook JS. Adolescent life events as predictors of adult depression. Journal of Affective Disorders 2002; 68: 49-57.

28 Hammen C. Cognitive, life stress, and interpersonal approaches to a developmental psychopathology model of depression. Development and Psychopathology 1992; 4:189-206.

29 Rice F, Farold GT, Thapar A. Assessing the effects of age, sex and shared environment on the gene tic aetiology of depression in childhood and ado lescence. Journal Child Psychol Psychiatry 2002; 43: 1039-51.

30 Silberg J, Rutter M, Neale M, Eaves L. Genetic moderation of environmental risk for depression and anxiety in adolescent girls. $\mathrm{Br}$ Journal Psychi atry 2001; 179: 116-21.

31 Canetti L, Bachar E, Bonne O, Agid O, Lerer B, De-Nour AK, Shalev AY. The impact of parental death versus separation from parents on the men tal health of israeli adolescents. Comprehensive Psychiatry 2000; 41:360-8.

32 González-Forteza C, Palos PA, Tapia AJ. Estresres cotidianos familiares, sintomatologia depresiva e ideacíon suicida en adolescents mexicanos. Acta psquiát Psicol Amlat 1997; 43: 319-26.
33 Ostrander R, Weinfurt KP, Nay WR. The role of age, family support, and negative cognitions in the prediction of depressive symptoms. School Psychology Review 1998; 27(1): 127-38. 34.

34 Coutinho DS, Baptista MN, Morais PR. Depressão pós-parto: prevalência e correlação com o suporte social. Infanto - Revista Neuro-psiquiatria da infância e adolescência 2002; 10: 63-71.

35 Bandura A. Observational learning. In: Social foundations of thought and action. New Jersey: Prentice-Hall; 1996. p. 47-105.

36 Miller L, Kramer R, Warner V, Wickramaratne P, Weissman M. Intergenerational transmission of parental bonding among women. Journal Am. Acad. Child Adolesc. Psychiatry 1997; 8: 1134-9.

37 Miller L, Warner V, Wickramaratne P, Weissman M. Self-esteem and depression: ten year follow-up of mothers and offspring. Journal of affective disorders 1999; 52: 41-9.

38 Reti IM, Samuels JF, Eaton WW, Bienvenu OJ, Costa Jr PT, Nestadt G. Influences of parenting en normal personality traits. Psychiatry Research 2002; 111: 55-64.

39 Pearce CM, Martin G, Wood K. Sigificance of touch for perceptions of parenting and psycho logical adjustment among adolescents. Journal Am. Acad. Child adolesc. Psychiatry 1995; 34 : 160-7.

40 Narita T, Sato T, Hirano S, Gota M, Sakado K, Uehara T. Parental child-rearing behavior as me asured by the parental bonding instru-ment in a Japanese population: factor structure and relationship to a lifetime history of depres-sion. Journal of affective disorders 2000; 57: 229-234.

41 Mulder RT, Joyce PR, Cloninger CR. Temperament and early environment influence comorbi dity and personality disordes in major depression. Comprehensive Psychiatry 1994; 35: 225-33.

42 Rodgers B. Reported parental behaviour and adult affective symptoms. 1. Associations and moderating factors. Psychological Medicine 1996; 26: 51-61.

43 Duggan C, Sham P, Minne C, Lee A, Murray R. Quality of parenting and vulnerability to depression: results from a family study. Psychological Medicine 1998; 28: 185-91.

44 Rey JM. Perceptions of $\sim$ oor maternal care are associated with adolescent depression. Journal of affective disorders 1995; 34: 95-100.

45 Klimidis S, Minas IH, Ata AW, Atuar GW. Construct validation in adolescents of the brief cur rent form of the parental bonding instrument. Comprehensive Psychiatry 1992; 33: 378-83.

Recebido em 05/10/2004 Aprovado em 27/10/2004 